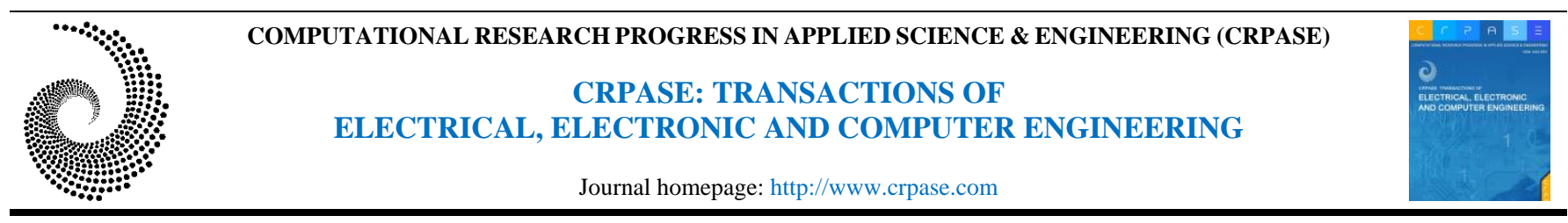

CRPASE: Transactions of Electrical, Electronic and Computer Engineering 7 (4) Article ID: 2411, 1-6, December 2021

ISSN 2423-4591

Research Article

\title{
Ultrasonic System to Improve Position Measurement and Shape Recognition Based on Neural Network
}

\author{
Siamak Hadadi * \\ Rahman Institute of Higher Education, Ramsar, Mazandaran, Iran
}

\begin{tabular}{ll}
\hline \multicolumn{1}{c}{ Keywords } & \multicolumn{1}{c}{ Abstract } \\
\cline { 2 - 4 } $\begin{array}{l}\text { Ultrasound, } \\
\text { Position recognition, } \\
\text { Shape recognition, } \\
\text { Time of flight, }\end{array}$ & $\begin{array}{l}\text { Today, with the advancement of technology, the use of remote detection systems in various } \\
\text { fields of medicine, industry and robotics has become common. In this regard, accurate } \\
\text { Neural network. }\end{array}$ \\
$\begin{array}{l}\text { recognition of the position and shape of objects is one of the main concerns. Guidance } \\
\text { systems for the blind, car parking, separation systems and intelligent robots are examples of } \\
\text { these systems. The basis of these systems is based on one of the methods of image } \\
\text { processing, infrared or ultrasound. In this paper, the researchers have dealt with a method } \\
\text { that has high diagnostic accuracy at a low cost and is resistant to environmental conditions. } \\
\text { The proposed method consists of two phases: position recognition and shape recognition. In } \\
\text { the first phase, the exact position of the object is calculated using the laws of wave physics } \\
\text { and the method known as the improved time of flight method. In the second phase, a set of } \\
\text { ultrasonic sensors with a two-dimensional matrix arrangement and a neural network were } \\
\text { used to detect the shape of the object. In the first phase, the average distance detection error } \\
\text { decreased from 1.875, which was related to the time of flight method, to } 0.271 . \text { In the second } \\
\text { phase, the efficiency of the neural network was } 96.8 \text { and the error rate was 3.2. The system } \\
\text { presented in both phases is very low cost and is associated with increased measurement } \\
\text { accuracy, which can therefore be a good alternative to other methods. }\end{array}$ \\
\hline
\end{tabular}

\section{Introduction}

Today, recognizing the shape and position of objects in various fields of science and technology is of great importance. For example: the production line of factories that want to separate objects with unusual appearance, Intelligent robots designed to scan environments where human presence is not possible due to adverse environmental conditions (Ecemis and Gaudiano 1999). [1]

There are similar conditions in medicine. For example, according to published statistics, there are about 39 million blind and more than 246 million low vision in the world (Aziz, Mahamad et al. 2008).[2]
In such a situation, one of the needs of medical science is to have a diagnostic tool to help the blind, something more than a white cane that is reliable and has high diagnostic accuracy, a tool that is also economically viable. Therefore, researchers have always sought to provide a diagnostic tool that is efficient and can calculate the exact position and shape of objects. many efforts have been made in this field, which has brought good results.

Generally, the basis of existing remote sensing systems is based on one of the methods of using infrared sensors (Zappi, Farella et al. 2010)[3], image processing (Rege, Memane et al. 2013) [4] or ultrasonic sensors (Cancela, Reyes et al. 2003)[5].

* Corresponding Author:Siamak Hadadi

E-mail address: hadadi@rahman.ac.ir

Received: 26 August 2021; Revised: 12 November 2021; Accepted: 12 December 2021

https://doi.org/10.52547/crpase.7.4.2411

Academic Editor: Noorbakhsh Amiri Golilarz

Please cite this article as: S. Hadadi, Ultrasonic system to improve position measurement and shape recognition based on neural network, Computational Research Progress in Applied Science \& Engineering, CRPASE: Transactions of Electrical, Electronic and Computer Engineering 7 (2021) 1-6, Article ID: 2411. 
The first method is simple and inexpensive, but their diagnostic accuracy is very low. Image processing methods are usually highly accurate, but the cost is much higher and in case of changes in environmental conditions, they face challenges or their accuracy decreases. For example, changing ambient light causes noise in the operation of these systems.

In the third method, ultrasonic waves are used to detect the shape and position of objects, which is low cost and has a high potential to improve measurement accuracy and increase detection power. In recent years, many attempts have been made to use ultrasonic sensors to detect the position and shape of objects, each of which has its advantages and disadvantages. For example, the researchers built and tested an ultrasonic belt to detect objects. The belt was equipped with three ultrasonic sensors.

They used time of flight position detection method to implement their proposed system. It can be said that their method was one of the first practical and operational implementations of ultrasonic waves to detect objects, the proposed system lacked the ability to detect the shape of obstacles and was not resistant to environmental conditions and noise (Probert, Lee et al. 1996)[6].

With the advent of new computational methods such as neural networks and genetic algorithms, researchers began to use these methods to achieve higher diagnostic clarity (Feizbahr, Mahdi et al. 2021) [7] .

Researchers in 2002 proposed a method for recognizing the shape of a cylinder based on a one-dimensional array of ultrasonic sensors and a genetic algorithm, the results of their experiments were promising (Ohtani and Baba 2002)[8].

Two years later, they developed their work and introduced a 3D diagnostic system based on a genetic algorithm and the rotation of a one-dimensional array of ultrasonic sensors around a target object. The results of these experiments were more favorable in the research dimension than the previous method, but due to the rotation of the sensor array around the cylinder, it had limited practical implementation and by changing the dimensions of the target object, the rotation radius also increased (Baba, Ohtani et al. 2004)[9].

However many problems in recognizing the position and shape of objects still remain. These problems include: Accuracy, reliability, resistance to environmental conditions and cost-effectiveness. Therefore, we proposed an ultrasonic diagnostic system to identify the position and shape of objects such as cylinders and cubes.

\section{Methods}

Figure 1 provides an overview of the proposed system. The system consists of an array of ultrasonic receiver and transmitter sensors with a matrix arrangement, a temperature sensor, a signal calculation and processing unit, and a detection unit.

The input unit consists of an ultrasonic sensor array with a two-dimensional matrix arrangement of transmitter / receiver sensors, and a temperature sensor. The processing unit is responsible for processing the raw input obtained from the sensors of the input unit. The output information of the receiver sensors will be provided to this unit for processing and performing calculations. After that, the information obtained from the processing unit will be provided to the detection unit as input. In this unit, the position detection and its final confirmation will be done in MATLAB software and the object shape will be identified by a multilayer neural network. The block diagram of the proposed system is shown in the figure below.

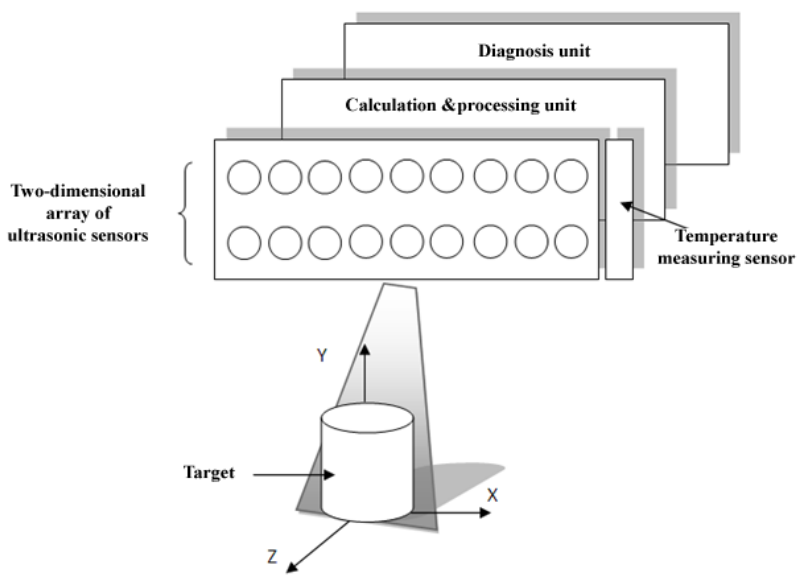

Figure 1. overview of the proposed system

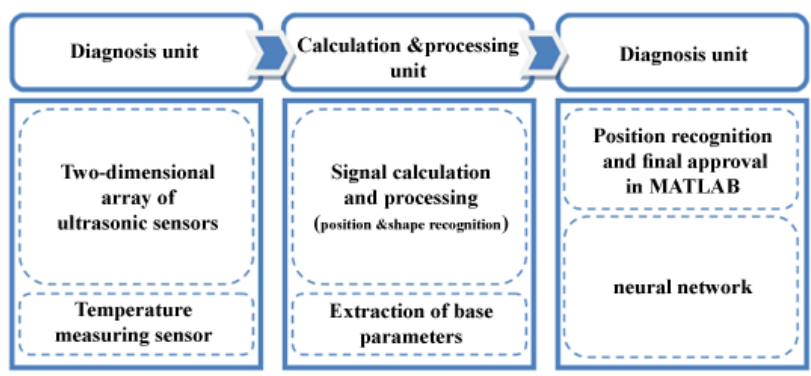

Figure 2. Proposed system block diagram

The basic principle in the proposed system is based on optimizing the time of flight method. Transmitter sensors detect 8 ultrasonic pulses with the selected optimal frequency in a specified time interval. When ultrasonic waves hit the target object, the reflection of the waves is received by the array of receiver sensors. By measuring the return pulse width and additional calculations, the required information is obtained and provided to the diagnostic unit. Then, in the first phase, the exact position of the object is calculated and in the second phase, information is provided to the neural network to detect the shape of the object.

\subsection{Position Measurement}

In the proposed system, the position of the target object is done in $3 \mathrm{D}$ space and in accordance with the $\mathrm{X}-\mathrm{Y}-\mathrm{Z}$ axes. The X-Y coordinate system is defined in parallel with the array of ultrasonic sensors and sensor number 5 has been selected as the base and origin sensor of the coordinates. This sensor is marked in black in Figure 4. In these experiments, the base sensor always calculates the depth of the object in the $\mathrm{X}-\mathrm{Z}$ coordinate system. 


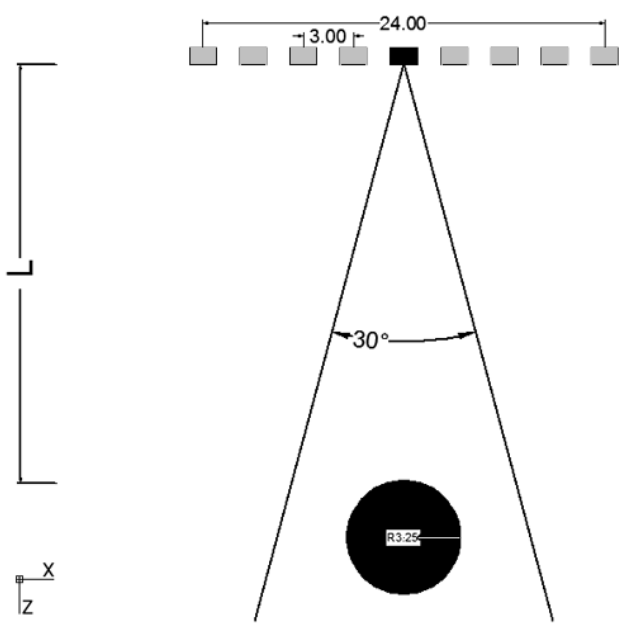

Figure 3. Position measurement in the proposed system

Achieving accuracy in calculating object depth is challenging. Factors influencing accuracy are: Existence of environmental noise, Characteristics of the reflective surface of an object such as the presence of an angle, Surface roughness, Temperature effects, Air pressure and relative humidity.

\subsection{Shape Recognition}

Shape detection in the proposed system is based on the distribution of the return pulse width of the array of ultrasonic receiver sensors and the calculation of the difference between the results obtained from the sensors.

In the experiments, the geometric shapes of cylinders and cubes with different dimensions were selected as the target object, which were placed at a constant distance from the array of receiver sensors. Figure 5 shows the radiation of ultrasonic waves to a cylinder with a radius of $3.25 \mathrm{~cm}$, located $25 \mathrm{~cm}$ from the array of sensors. In this image, the radiation angle is equal to 30 degrees. The dashed areas indicate the existence of an overlap phenomenon. In this case, in addition to the influencing factors mentioned earlier, avoiding the phenomenon of overlap is a new challenge in shape recognition. The results obtained from the array of receiver sensors after processing and performing calculations will be provided to the neural network for shape recognition.

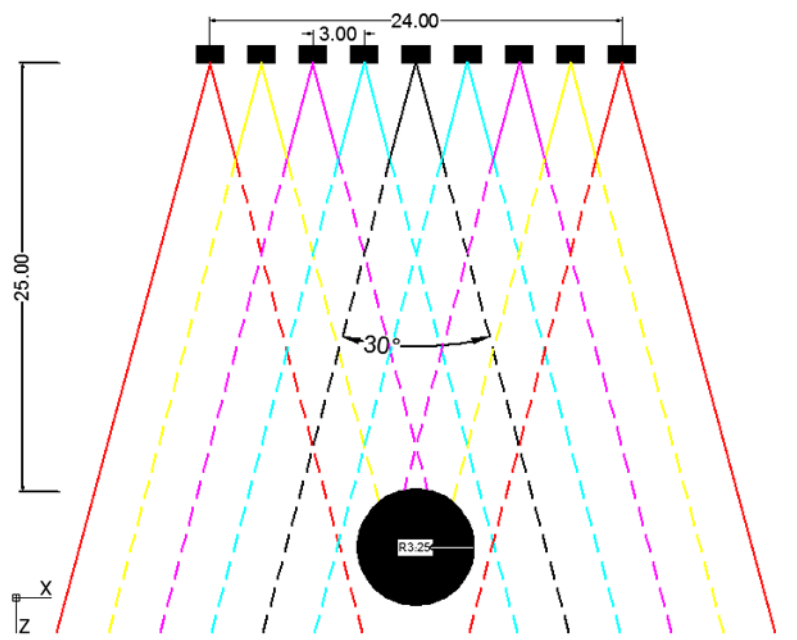

Figure 4. Ultrasonic radiation to a cylinder with a radius of $3.25 \mathrm{~cm}$

\section{Conditions and Configuration}

\subsection{Noise}

Since ultrasonic waves are transmitted in a conical manner with a maximum angle of 45 degrees, the signals reflected from other objects as noise are effective in the results obtained from the sensors, so in order to reduce these effects a cylindrical coating with a length of $25 \mathrm{Mm}$ is connected to the transmitter sensor.

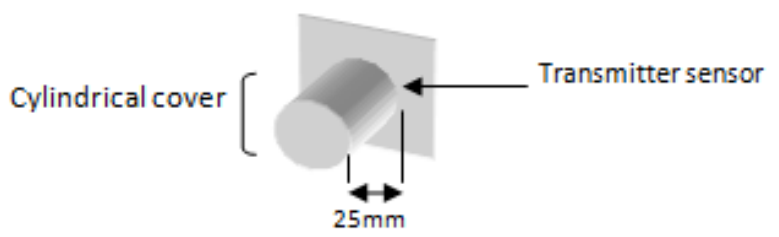

Figure 5. Cylindrical cover

\subsection{Superposition}

In order to avoid the effects of interference waves, sending and receiving ultrasonic waves by sensors are done serially. In the proposed system, each sensor sends and receives waves 12 times, which according to the information obtained from the datasheet, the maximum time of 36 milliseconds between each sending and receiving seems to be safe. The whole system consists of 18 sensors, so the time required to complete a complete cycle is 7776 milliseconds.

\subsection{Ultrasonic Sensors}

Choosing the operating frequency of ultrasonic sensors is a compromise between conflicting needs. High frequencies will be desirable to increase the resolution and enjoy high resolution of the system. However, with increasing frequency, the life of the waves decreases and as a result, the maximum detectable distance decreases.

Therefore, considering all the conditions, a relatively low frequency of $40 \mathrm{kHz}$ was selected as the operating frequency in the system. Taking into account the above and because of the low cost of the system, HSR 04 ultrasonic modules were selected for this purpose.

Features of this sensor module include very low cost, stable performance, operating power for measuring distances from 2 to $300 \mathrm{~cm}$ and having a suitable accuracy of $3 \mathrm{~mm}$, which works with only one source like a battery.

To start, a high-level pulse is sent to the trigger base for 10 microseconds, after which the module automatically sends 8 ultrasonic pulses at a frequency of $40 \mathrm{kHz}$. If the wave hits the obstacle and returns it to the sensor, the output of the echo base will be equal to the maximum value of 5 volts, which indicates the presence of an obstacle. By measuring the width of this return pulse, the distance to the obstacle can be calculated.

Figure 6 shows the arrangement of 18 HCSR-04 sensors in the ultrasonic sensor array. The hollow circles marked with the letter $\mathrm{T}$ in the image are the transmitters of ultrasonic waves and the hatched circles are the receivers of the waves. 


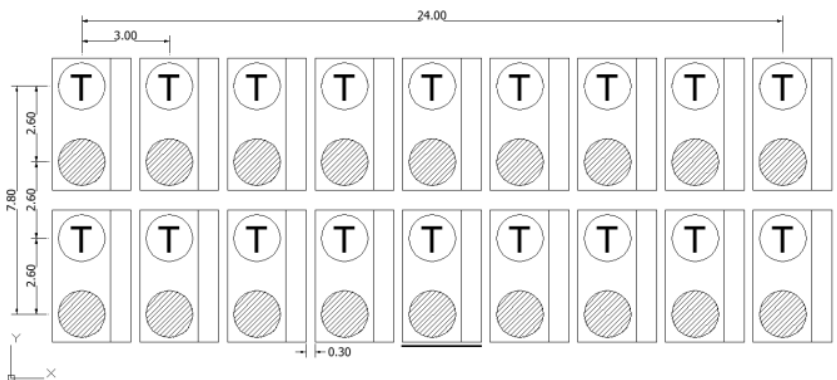

Figure 6. Array of ultrasonic sensors

\subsection{Temperature Control}

The time of flight method is based on the speed of sound in the air $(340 \mathrm{~m} / \mathrm{s})$ and this is while the air temperature has the greatest effect on the speed of sound, so achieving the desired accuracy without considering temperature changes will not be possible. Achieving the desired accuracy will not be possible without considering temperature changes. One of the methods to control temperature changes on the performance of ultrasonic sensors is to use a thermal compensation circuit.

In order to control the cost and availability of the system, the ultrasonic modules used do not have a thermal compensation circuit, so by adding an LM35 temperature sensor, we controlled the temperature changes at the programming level as shown below. Temperature and speed changes follow Formula 1, where $\mathrm{C}$ is the speed of sound, $\mathrm{T}$ is the temperature in degrees Celsius, and 331.30 is the speed of sound at $0^{\circ} \mathrm{C}$.

$$
C=331.30+0.606 T
$$

Therefore, in order to achieve the expected accuracy, we optimized the time of flight with the following equation.

$$
D=(331.30+0.606 T) t / 2
$$

Where $\mathrm{D}$ is the distance calculated and $\mathrm{t}$ is the time obtained from the sensor. Temperature changes have the greatest effect on the measurement accuracy of sensors and the speed of ultrasonic waves. Since the HCSR-04 sensors do not have a heat compensation circuit, we added an LM35 temperature sensor to the set of inlet unit sensors. High accuracy up to half a degree Celsius, Linear output for each degree of temperature change, measuring power and temperature tolerance from -50 to $+150{ }^{\circ} \mathrm{C}$ and low price led to the selection of this sensor.

\subsection{Signal Calculation and Processing unit}

This unit is responsible for receiving data from sensors, processing, calculating the position of the object and transmitting the obtained data to the detection unit. The processing unit is equipped with a Mega 2560 microcontroller.

\subsubsection{Diagnosis Unit}

The detection unit consists of two parts: position detection and shape detection.

\subsubsection{Position Detection}

In position detection, the distance of the object is calculated optimally.

\subsubsection{Shape Recognition}

In order to identify the pattern and geometric classification of the shapes, a multi-Layer Neural Networks with Sigmoid Function was used. That was learned by back propagation method. In these experiments, the neural network pattern recognition tool of MATLAB software was used. Obviously, the obtained results can be extended to the implementation of neural network hardware.

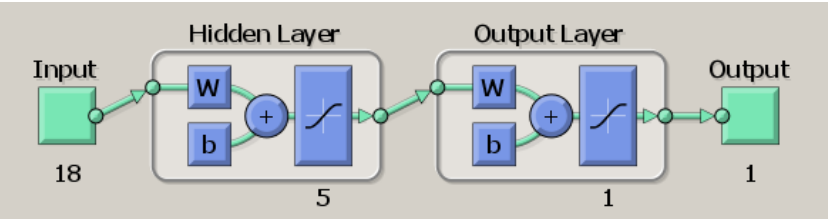

Figure 7. Schematic image of the proposed neural network

\section{Expriment}

In this study, two types of experiments were performed.

1- Measuring the position

2- Recognize the shape of an object with free width, constant depth and no angle

\subsection{Test Objects}

Cylinders and cubes in different dimensions were used. In type 1 experiments, a cylinder with a diameter of $6.5 \mathrm{~cm}$ was selected as the target object. In type 2 experiments, 31 cylinders and 31 cubes were used. The minimum diameter of the cylinders was 5 and the maximum was $20 \mathrm{~cm}$ and the minimum and maximum width of the cubic shapes were 5 and $20 \mathrm{~cm}$, respectively.

\subsection{Test Method}

The following are the test methods.

\subsubsection{Measure the Position}

This experiment was performed at different temperatures and distances in 3 steps, as follows:

A) The cylindrical object is placed at different distances from the base sensor, the actual distance from the sensor to the object is measured manually and we named this real distance D.

B) The base sensor measures the pulse width of the received signal, which is directly related to the return time and ambient temperature.

C) Using the LM35 sensor, the ambient temperature is calculated in degrees Celsius.

Using the obtained results, we calculate the distance once with the time of flight method (D1) and once with the optimized method (D2).

In MATLAB, the error percentage is calculated for each of the distances D1 and D2 to investigate whether the proposed method can lead to a reduction in the error percentage compared to the time of flight method. 


\subsubsection{Recognize the Shape of an Object with Free Width,} Constant Depth and no Angle

Shape recognition consists of 4 steps, which are discussed below.

A) Measuring objects are placed one by one at a fixed distance of $25 \mathrm{~cm}$ and without angles.

B) Each of the sensors sends and receives waves sequentially (according to what was said in the superposition) in a 12-step cycle. At this stage, a teaching model for the neural network is obtained.

C) The neural network is trained to recognize the shape of the object using the training pattern obtained from the previous step. Learning by error propagation method continues with the number of learning steps more than $1,000,000$ or by continuing the training until the learning error is less than 0.0001 .

D) A cylinder or cube with untrained dimensions is placed at a distance of $25 \mathrm{~cm}$ from the array of sensors and the shape of the object is detected using the obtained pattern.

\section{Result}

The results were presented in two sections: position measurement and shape recognition.

\subsection{Position Measurement}

Figure 8 shows the percentage of measurement error in the two methods studied in 15 experiments. The blue lines are related to the time of flight method and the red lines are related to our optimized method. The error rate in all experiments has been associated with improvement, and even in some experiments, such as Experiment No. 9, the error percentage has been reduced by more than $3 \%$, which is very significant.

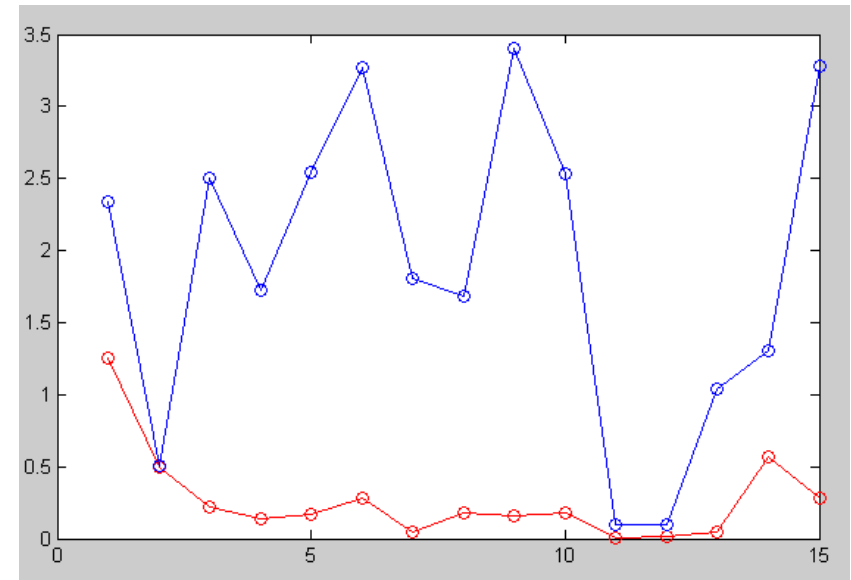

Figure 8. comparison of error percentage

\subsection{Shape Recognition}

In shape recognition, each sensor sends waves 12 times in a complete cycle. The results for each sensor are sorted in ascending order. In order to eliminate the possible consequences of noise in the environment, the first and last value is removed and then the average is taken from the other 10 numbers. For example, the diagram below shows the values obtained for each sensor in front of a cylinder with a diameter of $20 \mathrm{~cm}$. After that, 18 obtained parameters were provided to the neural network to detect the shape.

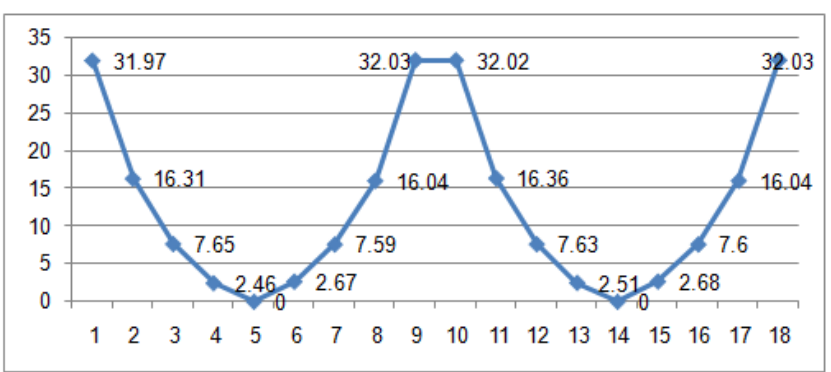

Figure 9. Shows the values of each sensor for a cylinder with a diameter of $20 \mathrm{~cm}$

In a similar way, the results obtained for a cube with a width of $20 \mathrm{~cm}$ are presented in figure 10 .

Data sharing in the neural network is very important to achieve scientific and principled results. We divided the data from 62 experiments into 3 categories: training data, test data, and validation data. In order to study the function of the neural network, we used a Confusion matrix, the results of which are presented in Figure 7. 29 samples from class number 1 (cube) and 31 samples from class number 2 (cylinder) have been correctly identified and the overall efficiency is equal to $96.8 \%$ and the total error percentage is equal to $3.2 \%$, which is a very desirable efficiency.

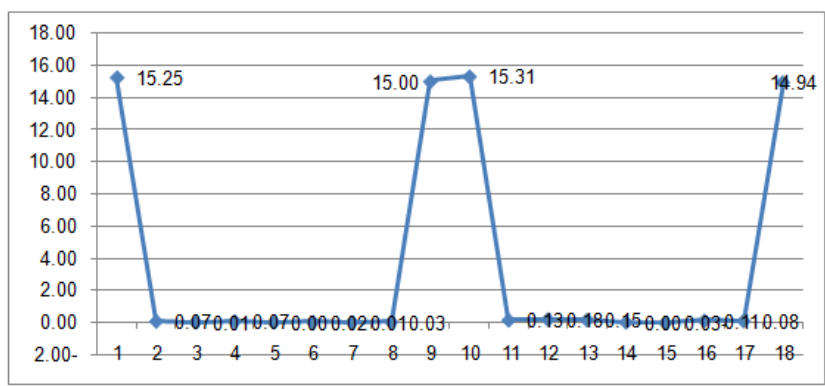

Figure 10. Results obtained for a cube
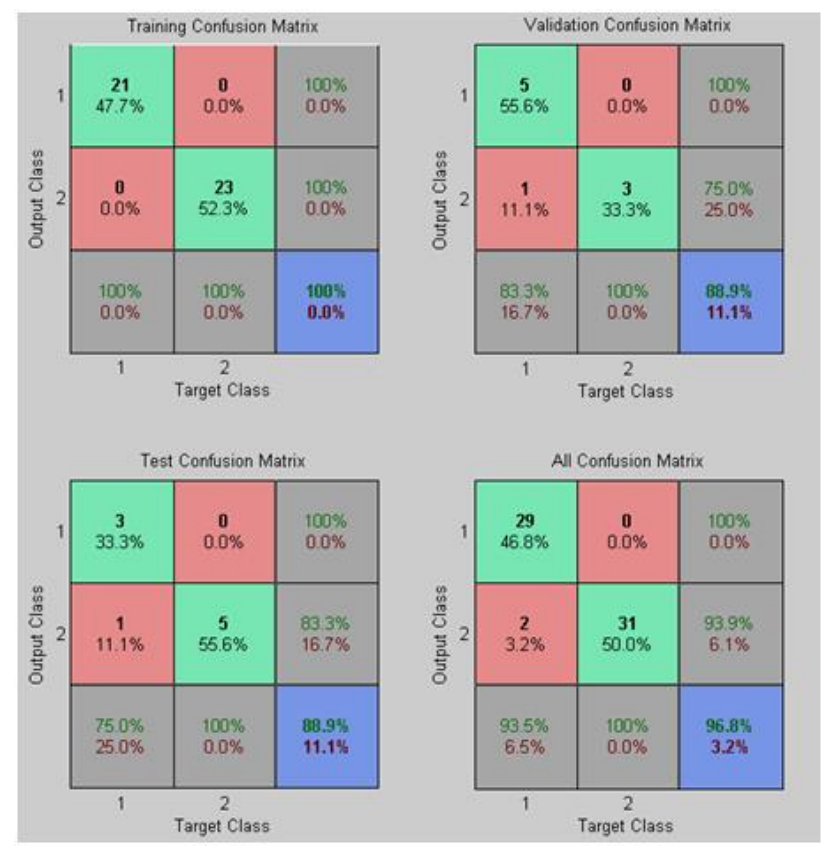

Figure 11. Confusion Matrix 


\section{Conclusion}

In Phase 1 of the proposed system, an optimal method of measuring position, cheap and resistant to temperature and noise was discussed. The results of the experiments showed that using this method, the average percentage of distance detection error in the time of flight method decreased from 1.875 to 0.271 . Therefore, the proposed method can be used in experiments that require double accuracy or in tools such as Guidance system for the blind and car parking. The results of the experiments In Phase 2 were very satisfactory, the percentage of neural network efficiency in shape recognition was 96.8 and the error rate was 3.2 , which is a very desirable efficiency. In addition to efficiency and reliability, the system presented in the second phase was also very low cost and economically viable. Although there are problems such as limited detection resolution in terms of width relative to the order and distance of the sensors from each other, the results of the experiments were very good and it was shown that the position and shape of objects can be detected with high resolution. With more study and changes in operating frequency, arrangement, distance, and number of ultrasonic sensors, more complex geometric objects can be identified.

\section{References}

[1] Ecemis, M. I. and P. Gaudiano, Object recognition with ultrasonic sensors. Proceedings 1999 IEEE International Symposium on Computational Intelligence in Robotics and Automation. CIRA'99 (Cat. No. 99EX375), IEEE (1999).
[2] A. Aziz, S. Mahamad, M. Mehat and N. Samiha, Blind echolocation using ultrasonic sensors, 2008 International Symposium on Information Technology (2008) 1-7.

[3] P. Zappi, E. Farella, L Benini, Tracking motion direction and distance with pyroelectric IR sensors, IEEE Sensors Journal 10 (2010) 1486-1494.

[4] S. Rege, R. Memane, M. Phatak, P. Agarwal, 2D geometric shape and color recognition using digital image processing, International journal of advanced research in electrical, electronics and instrumentation engineering 2 (2013) 24792487.

[5] P. Cancela, F. Reyes, P. Rodriguez, G. Randall and A. Fernandez, Automatic object detection using shape information in ultrasound images, Proceedings 2003 International Conference on Image Processing (Cat. No.03CH37429) (2003) III-417.

[6] Probert, P., D. Lee, G. Kao, Interfaces for multi-sensor systems for navigation for the blind, EDVR Conference on Assistive Technology, Reading (1996).

[7] Feizbahr, M., Tonekaboni, N., Jiang, G. J., \& Chen, H. X. Optimized Vegetation Density to Dissipate Energy of Flood Flow in Open Canals. Mathematical Problems in Engineering., (2021).

[8] Ohtani, K. and M. Baba, A new method for shape recognition of a pillar-like object using a 1-D ultrasonic sensor array and GA. Proceedings of the 41st SICE Annual Conference. SICE 2002., IEEE (2002).

[9] M. Baba, K. Ohtani and S. Komatsu, 3D shape recognition system by ultrasonic sensor array and genetic algorithms, Proceedings of the 21st IEEE Instrumentation and Measurement Technology Conference (IEEE Cat. No.04CH37510) (2004) 1948-1952. 